\title{
South African measles outbreak 2009 - 2010 as experienced by a paediatric hospital
}

David M le Roux, Stanzi M le Roux, James J Nuttall, Brian S Eley

Introduction. Between 2009 and 2010, South Africa experienced a major measles outbreak, with more than 18000 confirmed cases reported to the National Institute of Communicable Diseases.

Methods. We studied measles admissions during the outbreak to Red Cross War Memorial Children's Hospital, Cape Town, between 1 November 2009 and 31 July 2010. Factors associated with mortality were retrospectively identified from notification records and hospital admissions data. Multivariate logistic regression was used to investigate potential risk factors for death.

Results. In total, 1861 children were diagnosed with measles; $552(30 \%)$ were admitted to hospital. The most common reason for admission was pneumonia $(379(68 \%))$ and/or diarrhoea (262 (48\%)). The median age at admission was 7.36 months (interquartile range (IQR) 5.0 - 10.7). The median duration of admission was 4 days (IQR 2 - 6); total hospital admission time was 3746 days (10.3 child-years). HIV status was known in 404 (73\%) children: 39/400 (14\%) were HIV-infected. Eighteen children died ( $3 \%$ of all admissions); 15 (83\%) of them were less than 1 year old. In the regression model, HIV-infection (adjusted odds ratio (aOR) $7.55,95 \%$ confidence interval (CI) 2.27 - 25.12) and female sex (aOR 3.86, 95\% CI 1.26 - 11.84) were associated with higher odds of death.

Conclusions. There was a large paediatric admission burden during the 2009 - 2010 measles outbreak in Cape Town; young children were predominantly affected. HIV-infected children had a significantly higher case fatality.

S Afr Med J 2012;102(9):760-764. DOI:10.7196/SAMJ.5984
Infectious Diseases Unit, Department of Paediatrics and Child Health, Red Cross War Memorial Children's Hospital and University of Cape Town

David M le Roux, MB ChB, DTM\&H, FCPaed (SA), MMed (Paed), MPH

James J Nuttall, MB ChB, FCPaed (SA), DTM\&H

Brian S Eley, MB ChB, BSc, FCPaed (SA)

Department of Paediatrics and Child Health, Red Cross War Memorial Children's Hospital and University of Cape Town

Stanzi M le Roux, MB ChB, DCH
Measles vaccination has been very effective in reducing the global measles disease burden. However, outbreaks can occur when inadequate vaccination coverage allows accumulation of a sufficient number of susceptible individuals in a population. The estimated level of population immunity needed to prevent measles outbreaks is about $95 \% .{ }^{1}$ South Africa introduced routine measles vaccination in 1975 , and measles became a notifiable condition in 1979. In 1995, the current 2-dose strategy (routine vaccination at 9 and 18 months) was adopted. Case-based surveillance was introduced in 1998, with all suspected cases having blood or urine tested at the National Institute of Communicable Diseases (NICD). ${ }^{2}$

Accurate coverage of measles vaccination has been difficult to determine. In 2009, the South African Department of Health (DoH) 
estimated national coverage with routine measles vaccine to be $99 \%$, but the World Health Organization (WHO) and United Nations Children's Fund (UNICEF) coverage estimates were only $65 \%{ }^{3}$ In 2004, a survey in Western Cape Province found $79 \%$ of children to be 'fully vaccinated. ${ }^{4}$ In 2005 , another study estimated that $93 \%$ of children had received the first dose of measles vaccine, but only $60 \%$ a second dose. ${ }^{5}$ The authors concluded that routine coverage in the Western Cape was too low to prevent a measles outbreak.

The last major outbreak in South Africa occurred in 1992. In July 2009, a cluster of measles cases was detected in Tshwane; in August, the outbreak spread to Johannesburg, and then rapidly throughout the rest of the country. The first case in the Western Cape was confirmed in September 2009; there were 14 cases in October, and by November the outbreak was well established throughout the Cape Town metropole. ${ }^{6}$ We report the epidemiology of measles cases presenting to a single paediatric hospital in Cape Town.

\section{Methods}

Red Cross War Memorial Children's Hospital (RXH) is a 290-bed, level 3 academic hospital in Cape Town. During the outbreak, children with measles who needed admission were stabilised and admitted to the 'short stay ward', from which they were either discharged home, transferred to another hospital (mild disease), or admitted to the medical wards or Intensive Care Unit (ICU) at RXH (severe disease or other significant co-morbid disease).

All children diagnosed with measles at RXH between 1 November 2009 and 31 July 2010 were retrospectively identified from paper notification records, electronic hospital admission records, and the ICU database. Folders were reviewed and patients meeting the WHO clinical case definition were included. ${ }^{7}$ Suspected measles cases that tested negative for measles IgM antibodies were excluded. Data were captured onto a paper form and entered into an electronic database. Final discharge dates and re-admission dates were extracted from the city-wide hospital admission computer system; this includeD all the time spent in any hospital in the city.

All children were managed according to written hospital protocols. In-patient management of pneumonia included intravenous ampicillin, gentamicin and cloxacillin. Vitamin A was dosed according to WHO guidelines. ${ }^{7}$ During the outbreak, all children attending RXH who did not have clinical measles were given measles prophylaxis. Children older than 6 months were given a single dose of intramuscular measles vaccine (Rouvax, Sanofi Pasteur, France); children under 6 months and children with severe immunosuppression were given an intramuscular dose of human immunoglobulin (Intragam, National Bioproducts Institute, South Africa), according to the manufacturer's instructions.

HIV testing was performed when deemed clinically appropriate by the treating clinicians. Children $>18$ months old were considered HIV-infected if HIV antibodies were detected by enzyme-linked immunosorbent assay (ELISA) testing. Results were confirmed on a second specimen with a manual ELISA (Enzygnost Anti-HIV 1/2 Plus, Siemens Healthcare Diagnostic Products $\mathrm{GmbH}$, Marburg, Germany). Children $<18$ months old were considered HIV-infected with positive HIV DNA PCR (Cobas Ampliprep system, Roche Molecular Systems, Branchburg, New Jersey, USA). Positive HIV DNA PCR results were confirmed with HIV viral load testing.

For summary statistics, categorical data are expressed as number (percentage); continuous data are expressed as median (inter-quartile range (IQR)). Median values were compared with the Wilcoxon rank-sum test. Risk ratios were calculated to compare the proportion of children who died or were re-admitted by gender and HIV status.

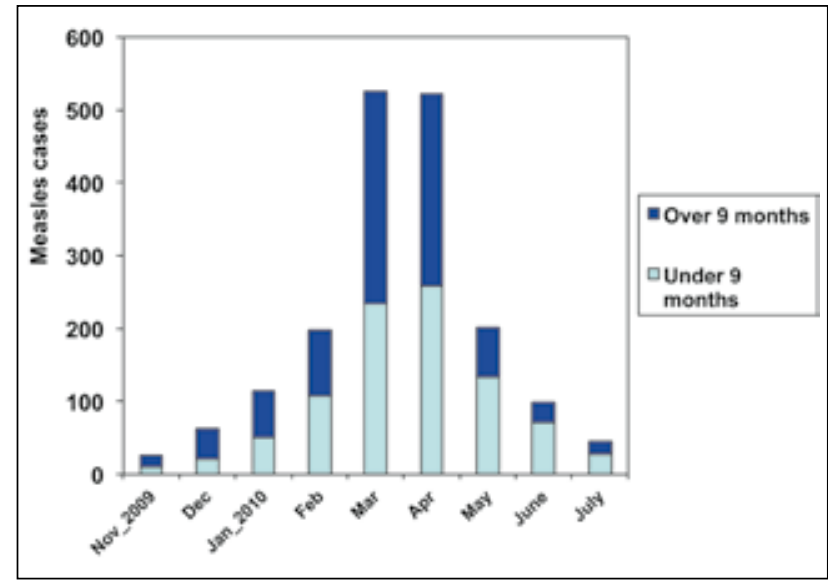

Fig. 1. Measles cases by month.

Factors associated with death were explored with multivariable logistic regression. We evaluated the influence of age, sex, HIV status, immunisation status, evidence of vitamin $\mathrm{A}$ administration, the presence of a 'Road to Health Card' and the weight-for-age Z-score (calculated from growth charts from the Centers for Disease Control and Prevention (CDC)). Weight-for-age Z-score of less than -2 was considered 'moderate underweight for age'; and less than -3 was considered 'severe underweight for age'. Variables associated with the outcome at the $\alpha=0.1$ level were included for multivariable analysis. Automated backwards and forwards step-wise model building procedures were used; potential models were compared with Akaike's Information Criteria (AIC). Collinearity was assessed with variance inflation factors. The final model chosen was most parsimonious and presented the best AIC value. A sensitivity analysis was done to examine the influence of the children with unknown HIV status. Analyses were done in Stata version 10 (Statacorp, College Station, Texas, USA). All $p$-values are two-tailed $(\alpha=0.05)$.

Ethics approval was obtained from the Department of Paediatrics and Child Health Departmental Research Committee and the University of Cape Town Human Research Ethics Committee, HREC-REF number 511/2010.

\section{Results}

From 1 November 2009 to 31 July 2010, 1861 children with measles presented to RXH, who have been categorised as follows.

\section{Outpatients}

In total, 1309 (70\%) children were treated as outpatients; most presented in March and April 2010 (Fig. 1). The median age was 8.9 months (IQR 6.1 - 30.4) (Table 1). Documentation of measles vaccination status in the hospital notes was poor: of the 565 children eligible for routine measles vaccination, only 270 (48\%) had vaccination status recorded, and only $68 \%$ had vitamin A administration documented in the hospital notes (Table 1).

\section{Admissions}

There were 552 hospital admissions; inpatients were significantly younger than children treated only as outpatients (median age 7.4 months v. 8.9 months, Wilcoxon $p<0.0001$ ) (Table 1). Most children (357/552 (65\%)) were younger than 9 months (the age of routine measles vaccination) (Fig. 2). At least 379 (63\%) children presented with pneumonia, $262(47 \%)$ with severe diarrhoea and $163(30 \%)$ with both. Other reasons for admission included croup (37 (7\%)), and suspected meningitis $(2(0.4 \%))$. Vaccination status was recorded in the hospital notes in 92/195 (47\%) children older than 9 months; 
Table 1. Characteristics of children with measles treated as outpatients and admitted to hospital

\begin{tabular}{|c|c|c|c|}
\hline & $\begin{array}{l}\text { Outpatients } \\
n=1309\end{array}$ & $\begin{array}{l}\text { Inpatients } \\
n=552\end{array}$ & $\begin{array}{l}\text { Total } \\
N=1861\end{array}$ \\
\hline Age: months: median (IQR) & $8.9(6.1-30.4)$ & $7.36(5.0-10.7)$ & $8.3(5.7-21.9)$ \\
\hline Eligible for routine vaccination ( $>9$ months) & $565 / 1309(43 \%)$ & $195 / 552(35 \%)$ & $760 / 1861(41 \%)$ \\
\hline Vaccination status documented in hospital notes & $270 / 565(48 \%)$ & $92 / 195(47 \%)$ & $362 / 760(48 \%)$ \\
\hline At least 1 dose of measles vaccine documented in hospital notes & $125 / 270(46 \%)$ & $40 / 92(45 \%)$ & $165 / 362(46 \%)$ \\
\hline \multicolumn{4}{|l|}{ Vitamin A: } \\
\hline Given & $895(68 \%)$ & $522(95 \%)$ & $1447(78 \%)$ \\
\hline Not documented in hospital notes & $414(32 \%)$ & $30(5 \%)$ & $444(22 \%)$ \\
\hline Boys & $610(47 \%)$ & & $902(48 \%)$ \\
\hline Girls & $526(40 \%)$ & $292(53 \%)$ & $786(42 \%)$ \\
\hline Unknown & $174(12 \%)$ & $260(47 \%)$ & $174(9 \%)$ \\
\hline \multicolumn{4}{|l|}{ HIV status } \\
\hline Known HIV status & & $404 / 552(73 \%)$ & \\
\hline HIV uninfected & & $369 / 404(90 \%)$ & \\
\hline HIV infected & & $39 / 404(10 \%)$ & \\
\hline On ART & & $20 / 39(51 \%)$ & \\
\hline Weight-for-age Z-score & & -0.76 & \\
\hline (IQR) & & $(-1.73-0.24)$ & \\
\hline \multicolumn{4}{|l|}{ Road to Health Card seen } \\
\hline Yes & & $323(58 \%)$ & \\
\hline No & & $68(12 \%)$ & \\
\hline Not documented & & $161(30 \%)$ & \\
\hline \multicolumn{4}{|l|}{ Notified } \\
\hline Yes & & $382(69 \%)$ & \\
\hline No & & $139(25 \%)$ & \\
\hline Not documented & & $31(6 \%)$ & \\
\hline Confirmed cases (IgM) & & $96 / 552(17 \%)$ & \\
\hline Median length of stay: days (IQR) & & $4(2-6)$ & \\
\hline \multicolumn{4}{|l|}{ Outcome: } \\
\hline Died & & $18(3 \%)$ & \\
\hline Discharged home & & $354(64 \%)$ & \\
\hline Transferred out & & $179(33 \%)$ & \\
\hline Number readmitted (<30 days) & & $72 / 534(13 \%)$ & \\
\hline
\end{tabular}

\section{Table 2. Deaths by sex, age, HIV status and vaccination status}

\begin{tabular}{lc}
\hline & Total deaths/total children (\%) \\
\hline Age & \\
$\quad<9$ months & $12 / 357(3.4 \%)$ \\
$>9$ months & $6 / 195(3.1 \%)$ \\
Sex & \\
Male & $5 / 292(1.7 \%)$ \\
Female & $13 / 260(5.0 \%)$ \\
HIV status & \\
HIV-infected & $7 / 39(18.0 \%)$ \\
HIV uninfected & $11 / 365(3.0 \%)$ \\
Unknown & $0 / 148$ \\
Vaccination status & \\
Any measles vaccination & $1 / 93(1.1 \%)$ \\
Never vaccinated & $11 / 215(5.1 \%)$ \\
Unknown & $6 / 244(2.5 \%)$ \\
Total & $18 / 552(3.2 \%)$ \\
\end{tabular}

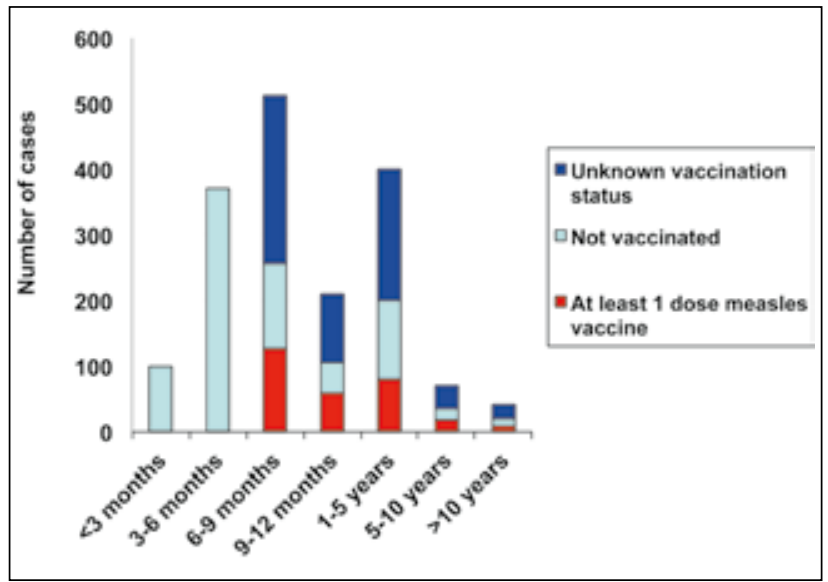

Fig. 2. Number of measles cases by age and vaccine status.

only 40/92 (45\%) had had any measles vaccine documented in the hospital notes (Fig. 2). During the study period, venous blood for serological testing was sent from 102 children admitted with 
Table 3. Odds ratios (unadjusted and adjusted) of death

\begin{tabular}{|c|c|c|c|c|}
\hline & Unadjusted OR (95\% CI) & $p$-value & Adjusted OR (95\% CI) & $p$ value \\
\hline Weight-for-age Z-score & $0.70(0.56-0.88)$ & 0.002 & $0.65(0.47-0.91)$ & 0.012 \\
\hline \multicolumn{5}{|l|}{ Sex } \\
\hline Male & 1 & & 1 & \\
\hline Female & $3.02(1.06-8.59)$ & 0.038 & $3.86(1.26-11.84)$ & 0.018 \\
\hline \multicolumn{5}{|l|}{ HIV status } \\
\hline HIV uninfected & 1 & & 1 & \\
\hline HIV infected & $7.04(2.55-19.41)$ & $<0.0001$ & $7.55(2.27-25.12)$ & 0.001 \\
\hline
\end{tabular}

suspected measles; 96 (17\%) had detectable measles IgM antibodies; 4 specimens were invalid or insufficient. Only 2 children with clinically-suspected measles were confirmed measles IgM negative; both children had detectable rubella IgM antibodies, and were excluded from this analysis.

Of the admitted children, 404 (73\%) had known HIV status: 39/404 (10\%) were HIV-infected, of whom 20/39 (51\%) had been on antiretroviral therapy (ART) for more than 3 months. All HIVinfected children with severe measles subsequently commenced ART. Only 3/23 (14\%) HIV-infected, vaccine-eligible children had documentation of any measles vaccination.

\section{Deaths and complications}

Of all admissions, 18 (3\%) children died, of whom 13 (72\%) were less than 1 year old; 11 (61\%) deaths were due to respiratory failure; other causes included septic shock with multi-organ failure (4 children (22\%)) and cerebral ischaemia after cardio-respiratory arrest (2 children (11\%)). One child died from massive bowel ischaemia and necrosis.

Of HIV-infected children 7/39 (18\%) died v. 11/365 (3\%) known HIV-uninfected children. Case fatality was $5 \%$ among girls v. $1.7 \%$ of boys (Table 2). In multivariable logistic regression adjusted for age and weight-for-age, HIV infection and gender remained strong predictors of mortality (Table 3 ). Of HIV-infected children who died, 2/7 (29\%) had received ART for at least 3 months.

During the study period, 4 children with complications of measles required tracheostomies ( $\mathrm{Sr}$ Jane Booth, personal communication). One child was referred with severe croup; 3 children developed upper airway damage following prolonged intubation and ventilation for severe pneumonia. All 4 entered the RXH home tracheostomy programme and were discharged home within 2 months of their admission but had recurrent re-admissions with respiratory infections. By March 2012, 3 children had been successfully decannulated, and one remains with a tracheostomy.

\section{Length of stay and re-admissions}

The median duration of admission was 4 days (IQR 2 - 6 days). HIVinfected children were admitted for longer (median 6 days, IQR 4 - 14 days) than HIV-uninfected children (median 3 days, IQR 2 - 5 days; Wilcoxon $p=0.0007$ ).

Among survivors, 53/287 (18\%) of boys were re-admitted v. 19/247 (8\%) of girls (risk ratio (RR) for re-admission 2.40 (95\% CI 1.46 - 3.94, $p=0.0003)$ ). Moderate or severe underweight for age (weight for age Z-score less than -2) was also strongly associated with readmission: of 531 surviving children with known weight-forage, $26 / 113(23 \%)$ who were moderately or severely underweight for age were re-admitted v. 46/418 (11\%) of children who were not underweight for age, RR 2.09 (95\% CI $1.36-3.23, p=0.0009$ ). Of HIV-infected children, $28 \%$ (9/32) were re-admitted v. $12 \%(44 / 354)$ HIV-uninfected children (RR 2.26, 95\% CI $1.22-4.20, p=0.01$ ).
Total primary hospital admission time was 3746 days (10.3 childyears). Of the 534 surviving children, 72 (13\%) were re-admitted within 90 days; 24 of these children (24/534 ( $4 \%$ of all the survivors)) were re-admitted a third time within 90 days of their first admission. The total time spent in hospital, including the primary admission and subsequent re-admissions within 3 months of discharge, was 4477 days (12.3 child-years). This constitutes $6.6 \%$ of total in-patient days at RXH during the study period.

\section{Discussion}

Between April 2009 and November 2010, South Africa experienced a national measles outbreak, with 18311 serologically confirmed measles cases. Almost a quarter of these cases were infants younger than 9 months. ${ }^{8}$ The magnitude was probably even greater, as many cases were diagnosed clinically: in our institution, only 96/552 (17\%) measles admissions were laboratory confirmed.

At $\mathrm{RXH}$, the main burden of disease, in terms of hospitalisations and mortality, was in infants $<1$ year old. There was an especially high burden in infants younger than 6 months. There are several reasons why the very young were so severely affected. Children become susceptible to measles infection once maternally-derived transplacental antibody levels are below a protective threshold. Vaccinated women transfer fewer antibodies than mothers whose immunity followed wild-type measles infection. In Belgium, babies of vaccinated women received a third of measles-specific antibodies v. babies born to women with immunity owing to natural measles infection. ${ }^{9}$ The antibodies are also lost more quickly: the median time to loss of immunity was 0.97 months for babies born to vaccinated women v. 3.78 months for babies born to women with previous measles infection. ${ }^{9}$

In Cape Town, there has been little circulating measles virus since the 1992 outbreak; most women of childbearing age have not had natural measles infection. Since its inception in 1975, the coverage of the measles immunisation programme has been too low; many women have never been vaccinated. Their babies received no measles-specific antibodies, rendering them susceptible to measles infection from a very young age.

Case fatality of measles can range from $0.1 \%$ in developed countries to $30 \%$ in outbreaks among refugee populations. The incidence is influenced by age, intensity of exposure to measles virus, nutritional status, vaccination status, immune deficiency, vitamin A administration, and access to appropriate case management. ${ }^{10}$ During the outbreak under review, case fatality among inpatients at RXH was $3 \%$ (18 deaths out of 552 admissions). Low weight-for-age Z-score was strongly associated with increased mortality, with $35 \%$ increased odds of death for every standard deviation below the expected weight for age. Furthermore, among survivors, low weight for age Z-score doubled the risk of re-admission. The finding of similar measles incidence among boys and girls, but with increased mortality among 
girls, has previously been described, ${ }^{11}$ but the biological mechanism is not well understood.

We observed increased case fatality among inpatients with HIVinfection. They had an $18 \%$ case fatality ratio, and 7 times higher odds of death than HIV-uninfected children, despite half of them being established on ART. This figure is higher than previously reported in areas with high HIV prevalence. In the 2003 - 2005 measles outbreak in Johannesburg, 14\% of known HIV-infected children died; they had a 3.3 times higher risk of death than HIVuninfected children. ${ }^{12}$ In a 6-year study in Zambia, case fatality among HIV-infected children was $12 \%$, with 2.5 times higher odds of death than HIV-uninfected children. ${ }^{13}$ Our higher case fatality may reflect a selection bias for disease severity, as children admitted to RXH had more severe disease than children treated as outpatients or in other facilities in the city.

For several reasons, HIV-infected children have a more severe clinical course than HIV-uninfected children. HIV-infected children have deficient cellular and humoral immunity and impaired innate immune responses. ${ }^{14}$ Despite commencing ART, HIV-infected children had twice the risk of re-admission v. HIV-uninfected children, which may reflect the combined immune-suppressive effect of acute measles infection in an already-compromised individual. HIV-infected children have suboptimal responses to measles vaccination. Vaccination, even if not fully protective against measles infection, can attenuate severity of disease. ${ }^{10}$ In HIV-uninfected children, response to measles vaccination is age-dependent, with better antibody responses in older children. At 9 months, about $90 \%$ will develop protective antibody levels (interquartile range $82-95 \%$ ); at 12 months, $99 \%$ will develop protective levels after a single dose of measles vaccine, (interquartile range $93-100 \%){ }^{7}$ However, the response in HIV-infected children to vaccination does not improve with age. A meta-analysis of 26 studies of vaccine responses in HIV-infected children at different ages showed highly variable responses, but no trend towards improved seroconversion among older children. The efficacy of a single dose of measles vaccine, whether given at 6,9 or 12 months, was about $59 \%$ (95\% CI $46-71 \%){ }^{15}$ These studies only reported attainment of protective antibody levels, and did not take into account T-cell mediated immunity, effectiveness of protection from clinical disease, or the effect of commencing ART. However, in a retrospective analysis of the 2003 - 2005 measles outbreak in Johannesburg, effectiveness of measles vaccine in preventing clinical disease among HIV-infected children was calculated at $63 \%{ }^{12}$ Infants born to HIV-infected mothers who are themselves uninfected (HIV-exposed but uninfected) also have increased susceptibility to measles infection. HIV-exposed infants have lower levels of maternal antibody, and protective antibody is lost earlier than in HIV unexposed infants. ${ }^{16}$

Among the children admitted to hospital, there were 69 possible 'vaccine failures' - children with a record of at least 1 dose of measles vaccine, who developed severe clinical disease requiring admission. There are several possible explanations for these vaccine failures. When vaccinations were documented, the admitting clinician noted whether measles vaccine had been given, but not the date of administration. It was not possible to determine retrospectively the age at vaccination or time from vaccination till clinical disease. All children $>6$ months old received measles vaccine if they attended hospital, and there were 2 nationwide mass vaccination campaigns (12 - 23 April and 24 - 28 May 2010). However, vaccination of young children is less effective than of children $>1$ year old. ${ }^{7}$ Some children might already have been infected with measles, but were vaccinated while in the incubation period. All children $<6$ months old seen at the hospital who did not have measles were given measles immune globulin. If these children were subsequently vaccinated, the immune globulin could impair vaccine effectiveness for up to 9 months. ${ }^{17}$ If they developed clinical measles despite having had a vaccine dose recorded in their Road to Health Card, they would be considered vaccine failures.

This study has several limitations. The data were captured retrospectively from clinical case notes, much data had not been recorded, vaccine status was very poorly recorded, and we were not able to explore many of the possible vaccine failures. Weight-forheight is a better marker of malnutrition than weight-for-age, but few children had their height measured. Some children with low weight-for-age Z-scores might have been born pre-term, which might be a marker of low birthweight or pre-term delivery, not adequacy of post-natal nutrition. Most of the clinically diagnosed cases were not laboratory-confirmed. Dermatologists reviewing children admitted with severe measles at another hospital in Cape Town during the epidemic found a high correlation between clinically-suspected measles and laboratory-confirmed measles. However, this might not apply to clinical diagnosis of mild disease in outpatients. ${ }^{18}$

\section{Conclusion}

The 2009 - 2010 measles outbreak was associated with a large case-load, and high morbidity and mortality at RXH; $>65 \%$ of the admissions were for children aged $<9$ months. Measles is a preventable disease, given sufficient political will for the interruption of measles transmission. Vaccine coverage must urgently be improved and supplementary immunisation activities to underserviced communities be targeted. HIV-infected children had significantly higher case fatality and risk of re-admission. Prevention of vertical transmission of HIV is a national priority, coverage of these programmes must be extended, and early infant HIV diagnosis and treatment should be improved.

Acknowledgements. We thank data capturers Ida Oliphant, Santie Horn, Spasina King, Margaretha Prins and Busi Skosana; and Jane Booth for information regarding the tracheostomy and home ventilation programme.

\section{References}

1. Moss WJ, Griffin DE. Measles. Lancet 2012;379:153-164.

2. Uzicanin A, Eggers R, Webb E, et al. Impact of the 1996-1997 supplementary measles vaccination ampaigns in South Africa. Int J Epidemiol 2002;31:968-976.

3. World Health Organization: Vaccine Preventable Diseases Monitoring System 2011. http://apps.who. int/immunization_monitoring/en/globalsummary/countryprofileresult.cfm 2010 (accessed 15 July 2011).

Moodley S. Immunisation coverage: Western Cape: Review of the data January 2001 - 2004. Cape Town: Provincial Government of the Western Cape, 2004.

Corrigall I, Coetzee D, Cameron N. Is the Western Cape at risk of an outbreak of preventable childhood diseases? Lessons from an evaluation of routine immunisation coverage. S Afr Med J 2008:98:41-45. diseases? Lessons from an evaluation of routine inm Bultin 2009:7:15 July 2011.

6. NICD NHLS. Communicable Diseases Surveillance Bulletin 2009;7:15 July 2011. World Health Organization. Measles vaccines: WHO position paper. Wkly Epidemiol Rec 2009;84:349-

8. NICD NHLS. Communicable Diseases Communique. December 2010;9

9. Leuridan E, Hens N, Hutse V, Ieven M, Aerts M, Van Damme P. Early waning of maternal measles antibodies in era of measles elimination: longitudinal study. BMJ 2010;340:c1626.

10. Cairns KL, Nandy R, Grais RF. Challenges in measuring measles case fatality ratios in settings without vital registration. Emerg Themes Epidemiol 2010;7:4

11. Garenne M. Sex differences in measles mortality: a world review. Int J Epidemiol 1994;23:632-642.

12. McMorrow ML, Gebremedhin G, van den Heever J, et al. Measles outbreak in South Africa, 2003-2005. S Afr Med J 2009;99:314-319.

13. Moss WJ, Fisher C, Scott S, et al. HIV type 1 infection is a risk factor for mortality in hospitalized Zambian children with measles. Clin Infect Dis 2008;46:523-527.

14. Moss WJ, Cutts F, Griffin DE. Implications of the human immunodeficiency virus epidemic for control and eradication of measles. Clin Infect Dis 1999:29:106-112.

15. Scott P, Moss WJ, Gilani Z Low N. Measles vaccination in HIV-infected children: systematic review and meta-analysis of safety and immunogenicity. J Infect Dis 2011;204 Suppl 1:S164-78.

6. Scott S, Moss WJ, Cousens S, et al. The influence of HIV-1 exposure and infection on levels of passively
and acquired antibodies to measles virus in Zambian infants. Clin Infect Dis 2007;45:1417-1424.
act

17. American Academy of Pediatrics. Measles. In: Pickering LK, Baker CJ, Kimberlin DW, Long SS, eds. American Academy of Pediatrics. Measles. In: Pickering LK, Baker CJ, Kimberlin DW, Long SS, eds.
Red Book: 2009 Report of the Committee on Infectious Diseases. 28th ed. Elk Grove Village, IL: American Academy of Pediatrics, 2009:448

18. Tod G, Carrara H, Levin M, Todd G. Dermatological manifestations of measles infection in hospitalised paediatric patients observed in the 2009-2011 Western Cape epidemic. S Afr Med J 2012;102:356.

Accepted 18 June 2012. 